\title{
Evaluation de l'attractivité de Glossina palpalis palpalis vis-à-vis de l'homme et du porc dans la région de Daloa en Côte d'Ivoire
}

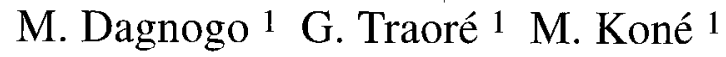

\section{Mots-clés}

Homme - Porcin - Glossina palpalis palpalis - Attractif - Piège - Variation saisonnière - Côte d'Ivoire.

\begin{abstract}
Résumé
Des expériences de captures de G. p. palpalis ont été faites en utilisant le porc et l'homme dans le village de Noumousséria II (zone forestière de Daloa) en Côte d'Ivoire. L'analyse des résultats montre que : 1) les récoltes faites avec le porc sont 2,4 fois plus abondantes que celles réalisées avec l'homme ; 2) le pourcentage de femelles capturées sur le porc esl plus élevé $(76,16 \rho .100)$ que celui des mâles alors que chez l'homme aucune différence n'apparaît entre les sexes ; 3) l'amplitude des variations saisonnières est plus marquée chez le porc que chez l'homme. Du point de vue qualitatif, une similitude a été observée entre les échantillons de glossines prises avec le porc et ceux du piège biconique. Malgré le faible nombre de glossines marquées recapturées, il semblerait que les mouches tsé-tsé reviennent plus au porc (12 mouches tsétsé) qu'à l'homme (aucune).
\end{abstract}

\section{INTRODUCTION}

Les glossines modifient leur comportement alimentaire selon la disponibilité des habitats en hôtes nourriciers. C'est ainsi que divers travaux ont montré le parallèle entre une forte présence de glossines et les villages où l'on trouve le porc domestique $(1,13$, $7,15)$. Une diminution brutale de cet animal pourrait donc amener, pendant un certain temps, les glossines à se rabattre sur l'homme. Ce risque devrait être pris cn considération car certains auteurs affirment que le porc est un réservoir du trypanosome humain $(9,19)$.

La présente étude a donc pour but d'analyser le comportement des mouches tsé-tsé marquées vis-à-vis de l'homme et du porc domestique.

\section{Lieu d'étude}

Il est situé dans la sous-préfecture de Daloa qui se trouve dans la région Centre-Ouest de la Côte d'Ivoire. Les essais ont eu lieu à Noumousséria II, village essentiellement peuplé par les ethnies Bétés et Niabouas. On y trouve, comme animaux domestiques, des porcs, des moutons et des chiens.

La zone forestière de Daloa a été touchée en 1940 par une importante épidémie de trypanosomose humaine qui y sévit depuis (8, $18)$.

\section{MATÉRIEL ET MÉTHODES}

Deux types de moyens de capture ont été utilisés pour la récolte des glossines : une enceinte en tulle moustiquaire avec une personne et le même système avec un porc comme appâts.

1. Centre universitaire de Formation en Entomologie médicale et vétérinaire (CEMV), 01 BP 2597 Bouaké 01, Côte d'Ivoire.

\section{Enceinte en tulle moustiquaire avec l'homme}

Le tulle blanc utilisé dans la confection de la moustiquaire est le même que celui du piège biconique. Les dimensions de la moustiquaire sont : $200 \mathrm{~cm}$ de longueur, $80 \mathrm{~cm}$ de largeur et $150 \mathrm{~cm}$ de hauteur.

Les bords inférieurs et le plafond sont en percale blanche. La moustiquaire, tenue sur quatre piquets en fer à béton, a les bords relevés de $10 \mathrm{~cm}$ au-dessus du sol pour permettre l'entrée des glossines.

Les mouches tsé-tsé qui entrent dans ce dispositif sont attirées tout d'abord vers la lumière du plafond où elles se réfugient ; l'homme sous la moustiquaire les récolte donc au fur ct à mesurc de lcur entrée à l'aide d'un tube à essai (figure 1).

\section{Enceinte en tulle moustiquaire avec le porc}

Les cages tombantes sont des dispositifs constitués d'un cadre métallique recouvert de tulle moustiquaire et suspendu à un arbre à $2 \mathrm{~m}$ au-dessus d'un appât animal. On abaisse la cage tombante à intervalles réguliers sur celui-ci. Les mouches tsé-tsé attirées par l'appât et enfermées dans ce dispositif sont alors capturées (10, 20 ).

La technique des cages tombantes a été modifiée et adaptée pour la première fois à la capture des mouches tsé-tsé en utilisant un dispositif fixe dans lequel se trouve une personne (6).

Par la suite, la capture sous moustiquaire avec une personne a été adaptée à différents animaux domestiques, dont le porc.

La cage pour animaux utilisée comprend une partie inférieure cubique de $1 \mathrm{~m}$ de côté et une partie supérieure conique qui supporte une cage apicale servant à recueillir les glossines. Le fer à béton de $8 \mathrm{~mm}$ de diamètre a été utilisé pour la confection des cages. La partie supérieure des cages est supportée par 11 barres soudées à la 


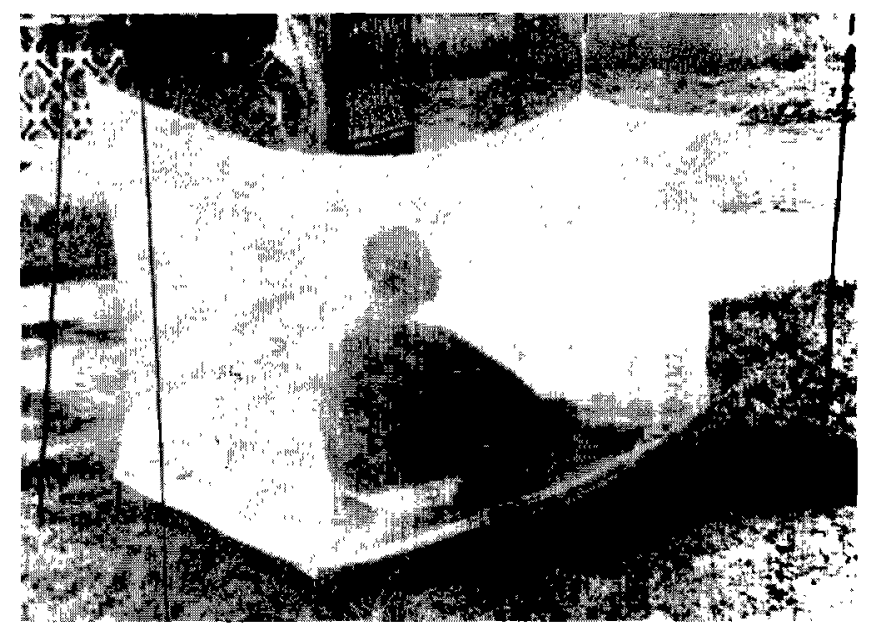

Figure 1 : échantillonnage de G. p. palpalis attirée par l'homme.

base et au sommet. Ce dispositif est entouré par le tulle moustiquaire blanc relevé de $10 \mathrm{~cm}$ au-dessus du sol pour permettre l'entrée des glossines (figure 2).

Dans cette étude, les cages pour animaux étaient visitées toutes les $30 \mathrm{~min}$ afin de récupérer toutes les glossines attirées par le porc. Ce temps relativement court permettait à l'observateur de récolter les mouches tsé-tsé, entrées dans ce dispositif, qui n'étaient pas encore montées jusqu'à la cage apicale. Les glossines étaient ainsi récoltées avant de se nourrir sur le porc.

Le porc dans le piège était arrosé trois fois par jour avec de l'eau pour éviter une très forte déshydratation.

\section{Marquage des glossines}

Les captures des glossines avec l'homme et avec le porc ont été faites en 1988 dans deux endroits différents, huit jours par mois, avec permutation chaque jour de la cage et de l'homme. Les glossines ainsi prises ont été marquées avec différentes couleurs de peinture gouache sur le scutum et relâchées aussitôt.

L'expérience a été faite à la périphérie du village de Noumousséria II chaque jour de 10 à $16 \mathrm{~h}$. Les glossines marquées recapturées ont été disséquées pour la recherche de trypanosomes.

\section{RÉSULTATS}

\section{Aspects quantitatifs}

Les différents moyens de capture ont permis de récolter 1229 glossines $G$. p. palpalis en 10 mois avec le porc comme appât et 519 avec l'homme.

La récolte des mouches tsé-tsé avec le porc $(12,29$ mouches/jour, tableau I) a donc été 2,4 fois plus importante que celle avec l'homme $\left(5,19\right.$ mouches/jour ; $\mathrm{Chi}^{2}=288,387 ;$ significatif à 0,001 pour $\mathrm{ddl}=1$ ).

Ces résultats sur la capture de $G$. p. palpalis avec le porc et avec l'homme sont comparables à ceux obtenus sur les préférences trophiques par analyse de l'origine du sang contenu dans le tube digestif des glossines. En effet, à Daloa les populations péridomestiques de $G$. p palpalis agressent 4 fois plus le porc (80 p. 100 des repas) que l'homme (5).

Le degré d'agressivité des glossines vis-à-vis du porc ou de l'homme est toutefois variable. Selon les auteurs, les résultats sur

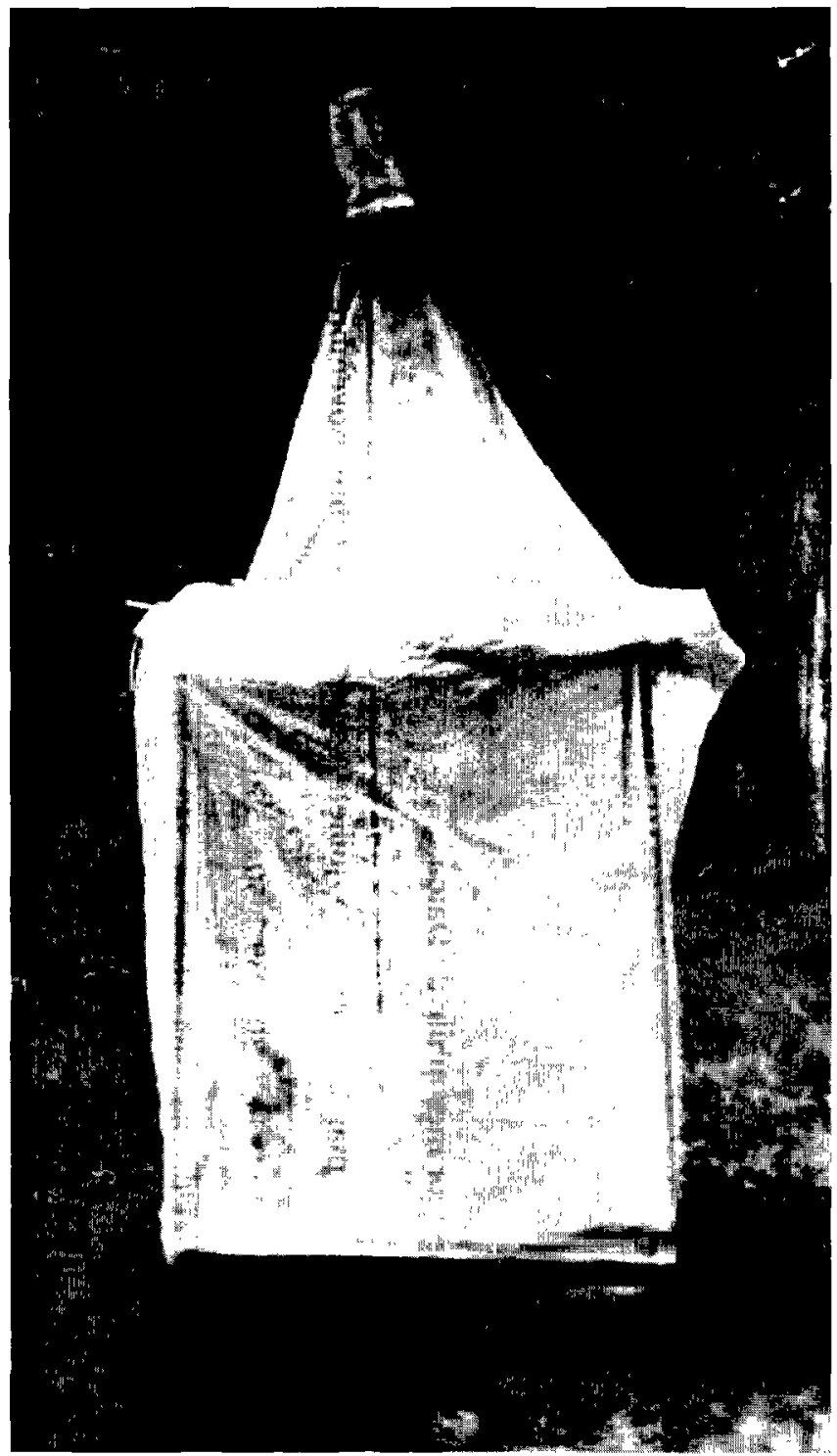

Figure 2 : échantillonnage de G. p. palpalis attirée par le porc.

les préférences trophiques des glossines pour le porc varient de 20 à 98 p. $100(1,15,13,7)$.

\section{Aspects qualitatifs}

Le rapport mâles/femelles (tableau I) de G. $p$. palpalis pris avcc l'homme (246 mâles, 273 femelles) est proche de 50 p. $100\left(\right.$ Chi $^{2}$ $=1,405 ;$ non significatif à 0,05 pour $\mathrm{dd} l=1$ ). Par contre le nombre de femelles de $G$. p. palpalis pris avec le porc (933) est 3,2 fois plus élevé que celui des mâles ( 296 ; Chi $=330,162$; significatif à 0,001 pour $\mathrm{ddl}=1$ ).

Ainsi les captures avec les pièges (biconiques, Vavoua) et avec le porc donnent-elles un pourcentage de femelles élevé $(3,16)$. Ces résultats rendent donc comparables ces deux méthodes de capture.

Selon plusieurs auteurs, le pourcentage élevé de capture des femelles dans une population correspond à la composition de la population naturelle car les femelles vivent en général plus longtemps que les mâles $(4,3)$. Les captures de $G$. p. palpalis avec l'homme ne correspondraient donc pas à la réalité.

Les résultats obtenus dans cette étude' s'expliqueraient soit parce que les mâles sont plus anthropophiles que les femelles soit parce 


\section{TABLEAU I}

Variations de la recapture de $G$. p. palpalis marquées

en utilisant le porc et l'homme comme appâts olfactifs (1988)

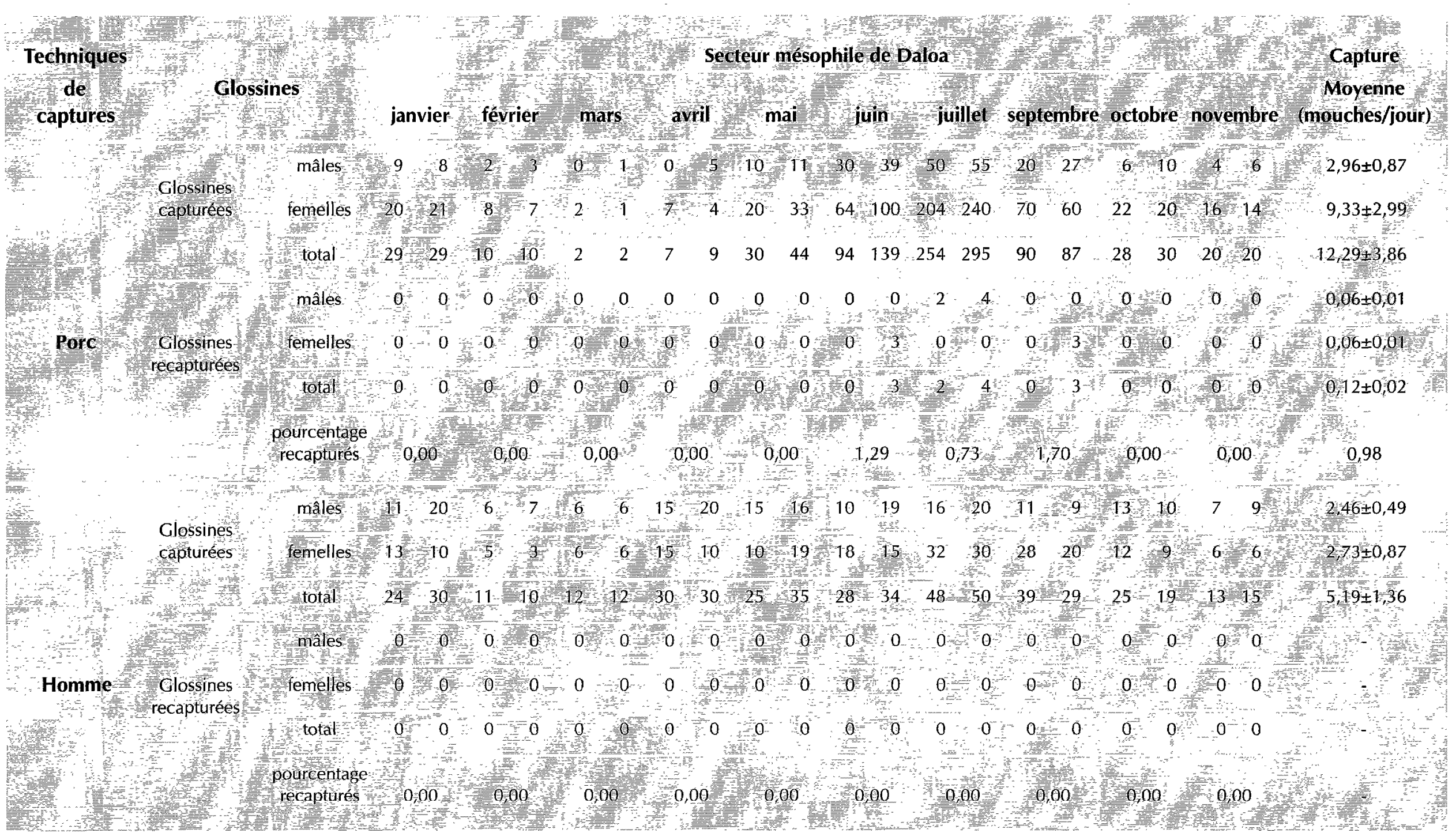


que ces dernières préfèrent se nourrir sur le porc. En effet, selon Gouteux et al. (12) les femelles acquerraient progressivement un comportement trophique qui les pousseraient à s'alimenter sur le porc.

\section{Variations saisonnières des captures avec le porc et avec l'homme}

La courbe des captures mensuelles avec le porc est bimodale avec un pic très prononcé en saison des pluies (juillet). A cette saison, le nombre de mouches tsé-tsé capturées avec le porc est en moyenne 10 à 13 fois plus élevé que celui de la saison sèche.

La courbe de la capture avec l'homme comme appât (figure 3) a une allure identique à celle faite avec le porc avec cependant de faibles amplitudes de variations saisonnières (pic étalé sur 5 mois). Cela explique les faibles coefficients d'asymétrie $(0,12)$ et d'aplatissement $(2,59)$ de la courbe de l'homme par rapport à ceux du porc (coefficients d'asymétrie 3,42 ; d'aplatissement 5,33 ).

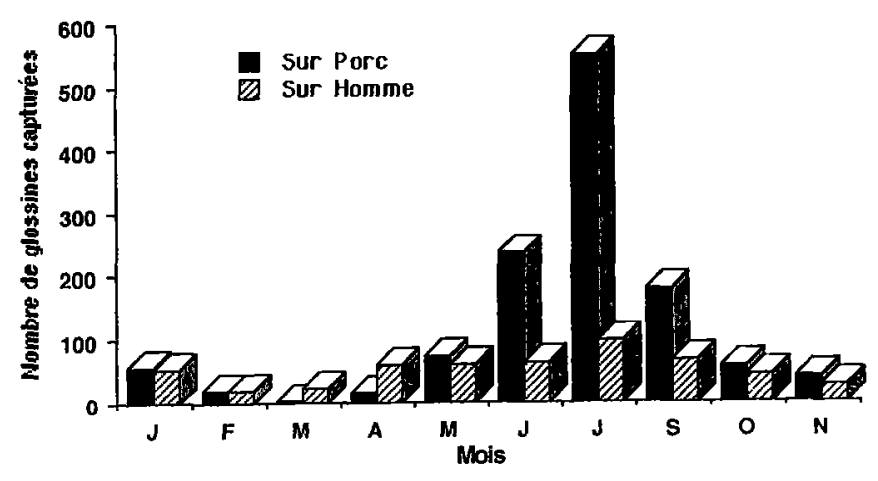

Figure 3 : variation du nombre de G. p. palpalis attirées soit par I'homme soit par le porc à la périphérie de villages de la région de Daloa (1988).

Selon plusieurs auteurs, la densité des mouches tsé-tsé crô̂t de la saison sèche à celle des pluies. Cette augmentation du nombre de glossines est liée à une amélioration des conditions climatiques qui induisent alors une plus grande longévité des glossines $(14,11$, 17).

L'utilisation du porc comme appât pour attirer les glossines rend mieux compte de cette augmentation.

\section{Taux de recapture des glossines marquées}

Sur 1229 mouches tsé-tsé capturées avec le porc en 10 mois marquées et relâchées, seulement 0,98 p. 100 sont revenues sur cet animal.

Par contre aucune mouche tsé-tsé des 519 prises avec l'homme n'a été recapturée. Aucune glossine prise sur le porc n'a été recapturée sur l'homme et vice versa (tableau I).

Ces résultats bien que préliminaires semblent indiquer que $G$. $p$. palpalis est plus attirée par le porc que l'homme.

Parmi les mouches tsé-tsé marquées recapturées avec le porc aucune différence liée au sexe ( 6 mâles, 6 femelles) n'a été observée.

Il ressort de l'analyse de ces résultats que les mouches tsé-tsé qui ont piqué le porc continuent toujours de s'alimenter sur le même animal. Dans ces conditions, on peut supposer que l'importance épidémiologique du porc comme réservoir de trypanosomes humains $(9,19)$ est alors amoindrie.

\section{CONCLUSION}

La capture de G. palpalis en utilisant le porc comme appât, à la différence de celle faite avec l'homme, donne des résultats comparables à ceux obtenus par le piège avec :

- une récolte abondante d'insectes ;

- un pourcentage de femelles très élevé ;

- des variations saisonnières nettes.

De plus, l'utilisation du porc permet l'étude simultanée de plusicurs espèces cocxistant dans les mêmes lieux, soit anthropophiles, soit zoophiles.

Le faible niveau de recapture des glossines marquées n'a pas permis d'apprécier l'amplitude des échanges entre les mouches tsétsé récoltées selon l'appât utilisé.

\section{Remerciements}

Les auteurs remercient très vivement Messieurs J. Mouchet (ORSTOM, Paris) et D. Cuisance (CIRAD-EMVT, Montpellier) ainsi que les deux lecteurs anonymes dont l'aide et les conseils ont fortement contribué à l'élaboration de cet article. Ce travail a bénéficié d'un appui financier du Programme spécial PNUD/Banque mondiale/OMS de recherches et de formation concernant les maladies tropicales.

\section{BIBLIOGRAPHIE}

1. BALDRY D.A.T., 1964. Observations on a close association between Glossina tachinoides and domestic pigs near Nsukka, Eastern Nigeria. II. Ecology and trypanosome infection rates in G. tachinoides. Ann. trop. Med. Parasit., 58: 32-34.

2. CHALLIER A., 1973. Ecologie de Glossina palpalis gambiensis Vanderplank, 1949 (Diptera : Muscidae) en savane d'Afrique Occidentale. Bondy, France, ORSTOM, 274 p. (Mém. ORSTOM N64)

3. CHALLIER A., EYRAUD M., LAFAYE A., LAVEISSIERE C., 1977. Amélioration du rendement du piège biconique pour glossines (Diptera : Glossinidac) par l'emploj d'un cônc inféricur blcu. Cah. ORSTOM, Sćr. Ent. méd. Parasitol., 15 : 283-286.

4. CHALLIER A., LAVEISSIERE C., 1973. Un nouveau piège pour la capture des glossines (Glossina, Diptera : Muscidae) description et essais sur le terrain. Cah. ORSTOM, Sér. Ent. méd. Parasitol., 11 : 251-262.

5. DAGNOGO M., 1994. Comportement dispersif et alimentaire de Glossina palpalis palpalis (Robineau - Desvoidy, 1830) (Diptera : Glossinidae) en relation avec la transmission de la trypanosomiase humaine africaine en Côte d'lvoire. Thèse doct. d'Etat, université d'Abidjan, Abidjan, Côte d'lvoire, 280 p. (N²04/94)

6. DAGNOGO M., LOHUIRIGNON K., EOUZAN J.P., 1987. Capture de Glossina palpalis palpalis (Robineau - Desvoidy, 1830) faite au piège biconique et sur captureur dans différents biotopes de la région de Daloa (Cote d'lvoire). Insect Sci. appl., $3: 323-326$.

7. DAGNOGO M., LOHUIRIGNON L., GOUTEUX J.P., 1985 Comportement alimentaire des populations péridomestiques de Clossina palpalis (Robineau - Desvoidy, 1830) et de Glossina tachinoides Westwood du domaine guinéen de Côte d'Ivoire. Cah. ORSTOM, Sér. Ent. mód. Parasitol., $23: 3-8$,

8. DOMERGUE-CLOAREC D., 1981. La lutte contre la trypanosomiase en Côte d'Ivoire, 1900-1945. J. Afr. History, $22: 63-72$.

9. GIBSON W., MEHLITZ D., LANHAM S.M., GODFREY D.G., 1978. The identification of Trypanosoma brucei gambiense in Liberian pigs and dogs by isoenzymes and by resistance to human plasma. Tropenmed. Parasit., 29 : 335-345. 
10. GOINY H.H., 1967. Man activated mobile bait trap for tsetse control and standardized density assessments of vector populations. Trans. $R$. Soc. trop. Med. Hyg., 61: 455-456.

11. GOUTEUX J.P., 1984. Ecologie des glossines en secteur préforestier de Côte d'Ivoire. Relation avec la trypanosomiase humaine et possibilité de lutte. Thèse doc. d'Etat, université Paris XI, Orsay, France, $450 \mathrm{p}$

12. GOUTEUX J.P., CHALLIER A., LAVEISSIERE C., 1981. Modifications et essais du piège à glossines (Diptera : Glossinidae) Challier Laveissière. Cah. ORSTOM, Sér. Lnt. méd. Parasitol., 21 : 07-99.

13. GOUTEUX J.P., LAVEISSIERE C., BOREHAM J.F.L., 1982. Ecologie des glossines en secteur préforestier de Côte d'Ivoire. 2. Les préférences trophiques de Glossina palpalis s.l. Cah. ORSTOM, Sér. Ent. méd. Parasitol., $20: 3-18$

14. GRUVEL J., 1974. Contribution à l'étude écologique de Glossina tachinoides (Westwood 1850) (Diptera : Muscidae) dans la réserve de Kalamaloué, vallée du Bas-Chari. Thèse doct. d'Etat ès-Sci. nat., université de Paris Vl, Paris, France, 302 p.

\section{Summary}

Dagnogo M., Traoré G., M. Koné. Glossina palpalis palpalis attractiveness testing towards humans and pigs in the area of Daloa in Côte d'Ivoire

Trapping experiments on Clossina palpalis palpalis were performed using humans and pigs as baits in the village of Noumousséria II (Daloa forest area, Côte d'Ivoire). The results show that: 1) the insect population collected with pig-baited traps was 2.4-fold larger than that with human-baited traps; 2 ) the percentage of females captured with pig-baited traps was higher $(76.16 \%)$ than that of males, while no significant difference between the two sexes appeared with humanbaited traps; 3) seasonal variations affected pig-baited traps more than human-baited traps. Qualitative analyses revealed that Glossina sampled from pigs were similar to those obtained with biconic traps. Despite the low number of dyemarked Glossina recaptured, it seems that tsetse flies are more attracted to pigs (12 tsetse flies) than to humans (none).

Key Words : Man - Swine - Glossina palpalis palpalis Attractant - Trap - Seasonal variation - Côte d'Ivoire.
15. LAVEISSIERE C., COURET D., STAAK C., HERVOUET J.P., 1985. Clossina palpalis et ses hôtes en secteur forestier de Côte d'Ivoire. Relations avec l'épidémiologie et la trypanosomiase humaine. Cah. ORSTOM, Sér. Ent. méd. Parasitol., 23 : 297-303.

16. LAVEISSIERE C., GREBAUT P., 1990. Recherches sur les pièges à glossines (Diptera : Glossinidae). Mise au point d'un modèle économique : le piège "Vavoua". Trop. Med. Parasitol., 41 : 185-192.

17. LAVEISSIERE C., HERVOUET J.P., 1991. La trypanosomiase humaine en Afrique de l'Ouest : épidémiologie et contrôle. Bondy, France, ORSTOM, $157 \mathrm{p}$

18. MEDA A.H., LAVEISSIERE C., DE MUYNCK A., DOUA F., DIALLO P.P., 1993. Les facteurs de risque de la trypanosomiase humaine dans les foyers endémiques de Côte d'Ivoire. Méd. trop., 1 : 83-92.

19. MEHLITZ D.. 1986. Le réservoir animal de la maladie du sommeil à Trypanosoma brucei gambiense. Maisons-Alfort, France, IEMVT, 156 p. (Etudes et Synthèses de l'IEMVT, N ${ }^{\circ} 18$ )

20. PHELPS R.J., 1968. A falling cage for sampling tsetse flies (Glossina : Diptera). Rhod. J. agric. Res., 6: 47-53.

Reçu le 18.12.95, accepté le 14.6.96

\section{Resumen}

Dagnogo M., Traoré G., M. Koné. Evaluación de la atracción de la Glossina palpalis palpalis con respecto al hombre y el cerdo en la región de Daloa, Côte d'Ivoire

Se realizaron estudios sobre las capturas de G. p. palpalis, en el cerdo y el hombre, en el pueblo de Noumousseria II (zona forestal de Daloa), en Côte d'lvoire. Los resultados obtenidos fueron los siguientes : 1) las colectas en el cerdo son 2,4 veces más ahundantes que las ohtenidas con el hombre; 2) el porcentaje de hembras capturadas sobre el cerdo es más elevado $(76,16$ p. 100) que el de machos, mientras que en el hombre no se observó ninguna diferencia entre los sexos ; 3) las amplitudes en las variaciones estacionales son más marcadas en el cerdo que en el hombre. Desde el purilo de vista cualitativo, se observó una similitud entre las muestras de glosinas capturadas con el cerdo y con la trampa bicónica. A pesar del bajo número de glosinas marcadas que se captuaron nuevamente, nuestras observaciones parecen indicar que las moscas tse-tse vuelven más al cerdo $(12$ moscas tse-tse) que al hombre (0).

Palabras clave : Hombre - Cerdo - Glossina palpalis palpalis Atrayente - Trampa - Variación estacional - Côte d'Ivoire. 